\title{
Poniendo en valor las papas nativas en el Perú
}

\author{
M. Ordinola ${ }^{1}$
}

\section{RESUMEN}

En el Perú, la papa es el principal cultivo de la sierra y de los pequeños productores, para quienes es una importante fuente de ingresos, alimento e incluso preservación de costumbres ancestrales. El Proyecto Innovación y Competitividad de la Papa (INCOPA) del Centro Internacional de la Papa (CIP) que se ejecuta con fondos de COSUDE y en alianza con una serie de socios públicos y privados, viene demostrando que la investigación y el desarrollo tienen que ir de la mano para obtener impactos efectivos a nivel de los pequeños productores. De manera operativa aplica el Enfoque Participativo de Cadenas Productivas (EPCP), que permite trabajar con los actores de la cadena de la papa y generar innovaciones que mejoren su competitividad. Los resultados alcanzados se refieren a: i) innovaciones comerciales o nuevos productos (papas nativas frescas seleccionadas, chips de papas de color, puré de papa amarilla, chuño blanco o tunta seleccionada); ii) innovaciones institucionales y nuevas normas (alianzas públicoprivadas, Día Nacional de la Papa, Ley de Comercio Mayorista de Papa, Norma Técnica de la Tunta, entre otros); y iii) innovaciones tecnológicas (manejo de post cosecha, producción de semilla de calidad, manejo integrado del cultivo, entre otros). La evidencia nos viene indicando la puesta en valor de las papas nativas de los pequeños productores y su revalorización como patrimonio cultural: su comercio posibilita que agricultores perciban precios $20 \%$ por encima de los canales comerciales tradicionales, un aumento del rendimiento de 10 a $14 \mathrm{tm} / \mathrm{ha}$ y una mejora de la calidad.

Palabras claves: cadenas productivas, innovación, mercadeo.

\section{Putting in value the native potatoes in Peru}

\begin{abstract}
In Peru, potato is the main crop in the Andean region and for small producers, for whom it is an important source of income and food, and also a way to preserve ancestral customs. The INCOPA Project (Innovation and Competitiveness of the Potato) of the International Potato Center (CIP), funded by the Swiss Agency for Development and Cooperation (SDC) in alliance with a series of public and private partners, is demonstrating that research and development can go hand in hand to obtain effective impact at the small producers level. The project effectively applies the Participatory Market Chain Approach (PMCA) that promotes working with the potato chain actors and R\&D organizations for linking research to market chain innovation. The objective is to improve the competitiveness of selected market chains and enable the effective participation of small-scale farmers. The results obtained refer to: i) commercial innovations or new products (selected fresh native potatoes, colored potato chips, yellow mashed potato and selected white chuño or tunta); ii) institutional innovations or new rules and norms (public-private alliances, National Potato Day, Potato Wholesale Commerce Law and the Tunta Technical Norm, among others); iii) technological innovations (post-harvest management, production of healthy seed and sustainable potato production technologies, among others). The evidence indicates that native potatoes' value and their appreciation as cultural heritage is essential for small producers. Their commercialization
\end{abstract}

${ }^{1}$ Coordinador Proyecto INCOPA, Centro Internacional de la Papa (CIP), Lima, Perú. cip-incopa@cgiar.org 
enables producers to obtain prices $20 \%$ above the prices offered by traditional channels, as well as better yield (from 10 to $14 \mathrm{tm} / \mathrm{ha}$ ) and quality improvement.

Key words: food production chains, innovation, marketing

\section{Introducción}

En la sierra peruana existe una amplia biodiversidad de la papa, que no es lo suficientemente aprovechada en términos sostenibles. Al mismo tiempo, la papa es el principal cultivo de los pequeños productores, para quienes es una importante fuente de ingresos, alimento e incluso, preservación de costumbres ancestrales.

Pero también es muy importante para la población urbana, porque este tubérculo milenario provee nutrientes y diversidad a la dieta diaria. Una limitación clave que tiene este sector, consiste en el escaso desarrollo comercial, ya que no se ha modernizado su imagen en fresco y tampoco se ha generado valor agregado en los últimos años.

En este contexto, uno de los aspectos claves a resolver es la generación de innovaciones que apoye el desarrollo competitivo del sector y promueva un trabajo conjunto y articulado de los actores a lo largo de la cadena productiva de la papa.

Los objetivos de este estudio fueron identificar los cambios que se vienen generando en el sector papa en el Perú, a partir de la intervención del Proyecto Innovación y Competitividad de la Papa (INCOPA) del Centro Internacional de la Papa (CIP) que se ejecuta con fondos de la Agencia Suiza para el Desarrollo y la Cooperación (COSUDE) conjuntamente con una serie de socios públicos y privados. Su trabajo se orienta a mejorar la competitividad de la cadena de papas nativas (desarrollo de nuevos productos, atracción de la empresa privada, mejoras en la eficiencia de los productores, articulación de cadenas productivas).

\section{Materiales y métodos}

De manera operativa aplica el Enfoque Participativo de Cadenas Productivas (EPCP), que se orienta a involucrar a todos los actores del proceso productivo del cultivo con el fin de generar innovaciones que mejoren la competitividad de la cadena productiva (Thiele y Bernet, 2005). INCOPA se implementa en las zonas de la sierra del Perú, teniendo un pequeño equipo de coordinación en Lima y a través de socios locales trabaja en las siguientes regiones: Ancash, Junín, Huánuco, Cajamarca, Cusco, Pasco, Ica, Huancavelica, Apurímac, Ayacucho y Puno.

\section{Resultados y discusión}

Como producto de este trabajo, se han logrado los siguientes resultados (Ordinola et al., 2009):

Innovaciones comerciales: son los cambios a nivel de productos finales que permiten mayor y mejor acceso de los pequeños productores a mercados dinámicos con mejor valor agregado. En esta línea se encuentran: "Mi Papa, Seleccionada \& Clasificada” (comercio mayorista), Chuño blanco (tunta) embolsado (mercado local y exportación); "Puré Andino" (exportación); “Tikapapa” (supermercados); Jalca Chips (exportación). Se debe indicar que tomando como punto de partida estas iniciativas se han lanzado recientemente al mercado nuevas marcas en base a las papas nativas (Lay's Andinas, Inca's Gold, Natu Krunch, Nips, Mr. Chips, entre otros).

Innovaciones institucionales: son los cambios en las reglas de juego en base a las cuales los agentes de la cadena y otros actores públicos se relacionan e interactúan. Pueden ser nuevas instituciones (CAPAC Perú, Alianza Institucional Tunta, Iniciativa Papas Andinas) o nuevas normas (Día Nacional de la Papa, Año 
Internacional de la Papa, Norma Técnica de la Tunta, Ley de Comercio Mayorista de Papa). Asimismo, un tema clave es la puesta en agenda pública (políticas) la necesidad de desarrollar de manera sostenible el sector papa en el Perú. Se debe remarcar que el Proyecto INCOPA trabaja con una amplia red de socios públicos y privados que permite ampliar la cobertura de las acciones y complementar acciones.

Innovaciones tecnológicas: son los cambios en tecnología requeridos para incrementar la eficiencia o la calidad de los procesos de producción y transformación en respuesta a demandas del mercado. Se pueden mencionar: normas y estándares de calidad de Mi Papa, inhibidores de brotes, difusión de estrategias de manejo integrado del cultivo, técnicas de almacenamiento, técnicas de producción de semilla.

La combinación de estos resultados tiene repercusión sobre los precios, las cantidades y los tamaños de los mercados de los productos de los pequeños productores.

\section{Conclusiones}

La evidencia nos viene indicando que el éxito comercial como alimento gourmet ha ayudado a demostrar el valor de las papas nativas de los pequeños productores y su revalorización como patrimonio cultural; su comercio posibilita que agricultores perciban precios $20 \%$ por encima de los canales comerciales tradicionales (combinando un aumento del rendimiento de 10 a $14 \mathrm{tm} / \mathrm{ha}$ y una mejora de la calidad); la experiencia ha estimulado el desarrollo de alianzas público-privadas $\mathrm{y}$ ha provocado importantes inversiones adicionales para el desarrollo de productos basados en la biodiversidad de la papa (Bucheli et al., 2007).

De manera general, se puede decir que el sector papa en el Perú - y en particular el segmento de papas amarillas y nativas- está en proceso de cambios. Como se ha podido ver, existen productos ya desarrollados por empresas privadas o productos nuevos que las empresas están investigando, porque los mercados así lo exigen. Para el continuo éxito de este proceso.

Implica que un mejoramiento en los ingresos de los productores de papa, es esencial que todos los actores en la cadena de valor compartan la visión común de vender productos de calidad, tanto frescos como procesados, para atender las demandas de mercado.

\section{Agradecimientos}

El autor quiere expresar su agradecimiento a la Agencia Suiza para el Desarrollo y la Cooperación (COSUDE) por su contribución al desarrollo de este trabajo.

\section{Literatura citada}

Bucheli, B., Ordinola, M., Antezana, I., Obregón, C., Maldonado, L. 2009. Estudio de caso: Evaluación de impacto de la intervención del Proyecto INCOPA/ADERS en Huánuco. Centro Internacional de la Papa (CIP). Documento de Trabajo 2009-2. Lima, Perú. 94 p.

Ordinola, M., Devaux, A., Manrique, K.,Fonseca, C., Thomann, A. 2009. Generando Innovaciones para el Desarrollo Competitivo de la Papa en el Perú. Centro Internacional de la Papa (CIP). Lima, Perú. 67 p.

Thiele, G., Bernet, T. (eds.). 2005. Conceptos, Pautas y Herramientas: Enfoque Participativo en Cadenas Productivas y Plataformas de Concertación. Proyecto Papa Andina, Centro Internacional de la Papa (CIP). Lima, Perú. 171 p. 